\title{
Competencias Docentes En El Uso Y Aplicación De Las Tic En San Juan Girón (Colombia)
}

\author{
Glenn Elmer Hernández Camelo \\ Profesor del Magisterio Nacional en Colombia y Doctorando \\ Ministerio de Educación Nacional de Colombia \\ Universidad de Granada/Facultad de Ciencias de la Educación \\ Dr. Juan Manuel Trujillo Torres \\ Profesor Titular de Universidad \\ Universidad de Granada. Facultad de Ciencias de la Educación \\ Departamento de Didáctica y Organización Escolar Campus de Cartuja s/n, \\ 18071, Granada, España
}

doi: 10.19044/esj.2016.v12n13p51 URL:http://dx.doi.org/10.19044/esj.2016.v12n13p51

\begin{abstract}
The study is part of a doctoral research on a model of teacher training to address approach the teaching process by educational technology, which aims to establish the percentage of teachers in the municipality of San Juan Giron (Colombia) with technology skills information and communication and how many of them apply them in the classroom. A questionnaire and an interview with principals, coordinators, teachers and students who are part of the public education system was applied. Data on correlation nonparametric tests, frequency tables and histograms and two-dimensional descriptive statistics in order to quantify the degree of association between variables analyzes were performed. The results relate to the percentages of skills in information and communication technologies which has the population studied, showing low rates of application of the same in the education sector and in the classroom. Among the most notable contributions it was found that the educational community is aware of the existence of technological tools but is not trained in the management of computing resources or the development of educational resources to support teaching because of the lack of effective plans educational and training models of teacher training, which has not allowed the pedagogical use of ICT in educational institutions. It is necessary to develop and implement a model of teacher training in ICT to respond to the educational reality of the schools in San Juan Giron.
\end{abstract}

Keywords: Teacher Training in ICT, Training Models, Educative Technology, Information and Communication Technologies 


\section{Resumen}

El estudio hace parte de una investigación doctoral sobre un modelo de formación de profesorado para abordar el proceso de enseñanza mediante la tecnología educativa, en el cual se pretende establecer el porcentaje de docentes del Municipio de San Juan Girón (Colombia) con competencias en tecnologías de información y comunicación y cuántos de éstos las aplican en el aula. Se aplicó un cuestionario y una entrevista a rectores, coordinadores, profesores y estudiantes que hacen parte del sistema educativo público. Sobre los datos se efectuaron pruebas no paramétricas de correlaciones, tablas de frecuencia e histogramas, así como estadística descriptiva bidimensional con el propósito de cuantificar el grado de asociación que existe entre las variables de análisis. Los resultados relacionan los porcentajes de las competencias en tecnologías de información y comunicación que posee la población estudiada, demostrando así bajos porcentajes de aplicación de las mismas en el sector educativo y en el aula. Dentro de las contribuciones mas destacables se encontró que la comunidad educativa tiene conocimiento de la existencia de herramientas tecnológicas pero no está capacitada en el manejo de recursos informáticos ni en la elaboración de materiale educativo de apoyo a la enseñanza debido a la carencia de planes efectivos de formación pedagógica y de modelos de formación docente, lo cual no ha permitido el uso pedagógico de las TIC en las instituciones educativas. Es necesario desarrollar y aplicar un modelo de formación docente en TIC que responda a la realidad educativa de las instituciones de San Juan Girón.

Palabras Clave: Formación Docente en TIC, Modelos de Formación, Tecnología Educativa, Tecnologías de Información y Comunicación

\section{Introducción}

El avance de las tecnologías de la información y comunicación y su aplicación en el sector educativo ha despertado, desde hace varios años, el interés de la comunidad académica mundial debido a que gracias a estas herramientas se pueden potenciar diversas habilidades en estudiantes y docentes propiciando así nuevos estilos de aprendizaje, nuevas metodologías de enseñanza y nuevos roles educativos que transforman a la sociedad. La inclusión de los medios tecnológicos en el sector educativo debe estar acompañada no sólo de la adquisición de nuevos y mejores equipos de computo y conectividad a redes de datos; debe estar soportada en nuevos esquemas de formación docente en TIC que permitan transformar la praxis educativa logrando así el uso pedagógico de estas herramientas en el aula. Tal como lo expresa Cabero y Batanero (2014) "las TIC nos pueden servir 
para ayudar a una serie de aspectos como son: poner en acción mejores o nuevos aprendizajes, establecer con ellas innovaciones pedagógicas y cambios organizacionales, facilitar los procesos de comunicación y la ruptura de la unidad de tiempo, espacio y acción, que es donde, por lo general, se desarrolla la acción formativa tradicional.” Al respecto, Fernandez, Hinojo y Aznar (2002) afirman que "la escuela no puede dejar de lado las nuevas tecnologías de la información y las comunicaciones, sino que debe preparar a las nuevas generaciones para convivir con estos medios promoviendo la participación y la reflexión crítica en su uso e interpretación, que a su vez, ha conllevado a la aparición de un gran reto: la formación del Profesorado en Tecnologías de la Información y la Comunicación”.

\section{La formación del profesorado en TIC:}

Uno de los aspectos más importantes en el momento de integrar las TIC en los procesos educativos es quizá la formación del profesorado. Este proceso debe caracterizarse por generar espacios educativos acordes que permitan a los profesores desarrollar competencias tecnológicas en torno al uso pedagógico de las TIC como herramientas de apoyo en el aula en las instituciones educativas. Trujillo y Raso (2010), al hilo, mencionan que "el futuro maestro deberá ser capaz de crear materiales curriculares, o de buscarlos, y generar su traslación didáctica, así como conocer la relación interdisciplinar entre las distintas áreas del currículo con el fin de presentar una visión globalizadora del conocimiento”. Ello implica necesariamente un cambio en el rol del profesorado, llevando a que éste se transforme en un generador de contenidos digitales y material educativo motivando así en sus estudiantes el uso adecuado de la tecnología para la construcción de competencias tecnológicas que les permita acceder a redes de conocimiento, seleccionar y clasificar la información, tal como lo menciona Trujillo (2006) “empieza a ejercer su tarea un profesorado comprometido con las TIC y su integración curricular con el fin último de facilitar el proceso de enseñanzaaprendizaje y mostrar al alumnado las posibilidades de aprendizaje más autónomo y motivador, entre otros factores”. Al respecto Quiroz (2012) afirma que "la inserción de las TIC en el ámbito educativo, implica un nuevo posicionamiento del profesor en su rol docente. Al introducirse las TIC, el profesor deja de ser el centro de la enseñanza y pasa a articular una nueva interacción entre los otros factores que interactúan en el quehacer educativo, los estudiantes, los materiales, los recursos y la información entre otros, dejando de ser la única fuente de información”. La generación de planes de formación docente en TIC debe ser el resultado de un análisis adecuado de las necesidades de formación que requieren los docentes, teniendo como eje principal el uso pedagógico de las herramientas tecnológicas y su incorporación al currículo, así como las características de la infraestructura 
tecnológica de las instituciones educativas. Lo anterior permitirá integrar estas tecnologías en los procesos educativos con el propósito de generar, clasificar, evaluar y compartir la información. Cabero (2015) manifiesta que "la solución no está exclusivamente en incorporar tecnologías, por muy adaptadas y cercanas que las mismas estén a los sujetos, sino también porque los sujetos hayan recibido una formación, alfabetización digital, que los capacite para obtener de las mismas el máximo provecho. Y esta formación desde mi punto de vista no se debe limitar a los aspectos meramente instrumentales, que llevan a que el usuario no sea una persona crítica en su utilización sino un mero consumidor pasivo de mensajes, sino a desarrollar la capacidad de localizar, evaluar, estructurar y organizar conceptualmente la información”.

\section{La tecnología educativa}

Del Moral (2010) afirma que: "Las TIC ofrecen nuevas posibilidades en el diseño y proceso de propuestas de enseñanza-aprendizaje como parte integral de la educación, que implican la adopción de nuevas metodologías de enseñanza que busquen potenciar el desarrollo cognitivo de los estudiantes”. Estas nuevas posibilidades han permitido la notoria evolución del conocimiento pedagógico sobre los medios y su influencia se ve reflejada no sólo en los nuevos planes de estudio de las instituciones educativas, sino que ha permitido a investigadores desarrollar nuevas áreas de estudio entorno a tópicos específicos que buscan integrar cada vez más de forma efectiva el uso de la tecnología en espacios educativos teniendo como referente las opiniones y experiencias del profesorado logrando así proponer nuevas metodologías que integren de forma adecuada las TIC en la enseñanza con el fin de transformar la educación, tal como lo expresa Adell (2010) "El concepto de Escuela 2.0, difuso e importado del campo de la tecnología e Internet, intenta recoger los retos y las oportunidades, y los deseos de cambio, de un creciente número de educadores y educadoras que ven en las nuevas tecnologías un elemento clave para transformar la educación y preparar a los jóvenes para afrontar los retos de esta nueva sociedad. También hay resistencias e intereses en que no cambie nada importante. Si triunfan, la brecha entre la sociedad y el sistema educativo será mayor aún”. De acuerdo a lo anterior, Trujillo y Raso (2010) afirman que "la formación del futuro maestro deberá centrarse en la resolución de problemas prácticos a través de la relación y la abstracción, que le permitan enfrentarse a situaciones similares a las que se va a encontrar en el mundo laboral y, de esta manera, contextualizar y construir los saberes a partir de tales situaciones". 


\section{Aportes de las TIC a la educación:}

Son diversos los aportes que desde las tecnologías de información y las comunicaciones se realizan al sector educativo. Algunos de ellos están centrados en el desarrollo de nuevas metodologías que permiten la adecuada utilización de herramientas digitales para desempeñar nuevas actividades y así atender diversas necesidades que en materia de formación exigen los estudiantes en el contexto tecnológico actual. Al respecto Del Moral (2010) sostiene que "en definitiva, el profesor 2.0 debe desempeñar nuevas tareas y poner en juego diversas competencias para atender a las necesidades formativas de los estudiantes en el contexto tecnológico actual, seleccionado y adaptando cada aplicación y herramienta Web 2.0 a las características de los alumnos, ofreciendo situaciones de aprendizaje mediadas por las TIC que favorezcan la adquisición de nuevos conocimientos”. Otro aporte importante es el relacionado con la posibilidad de acceder a la información desde cualquier parte del mundo lo cual mejora sustancialmente los procesos de enseñanza, propiciando la educación virtual, así como lo mencionan Aznar y Cáceres (2005) cuando afirman que las TIC brindan "la posibilidad de tener acceso a la información desde cualquier parte del planeta, eliminando fronteras, distancias; en el ámbito educativo y formativo, la posibilidad de hacer más cómoda la enseñanza-aprendizaje, mediante las aulas virtuales, a través de Internet, adaptándose a las características concretas de cada usuario, etc”. También, Trujillo, Morales, Ortiz y Raso (2015) proponen que "el cambio de metodología es un aspecto importante valorado por el alumnado, considerado como necesario para la adaptación a la sociedad actual, estando igualmente de acuerdo ante la introducción de las TIC en el proceso educativo, sin olvidar el componente de presencialidad de profesorado y alumnado en dicho proceso". Otro aporte significativo de la integración TIC en el ámbito educativo está relacionado con la utilización de las redes sociales como elemento formativo que permita desarrollar competencias que lleve al estudiante a explorar nuevas formas de interactuar con la virtualidad, tal como lo manifiesta Barroso y Cabero (2012) "dicho en otros términos, la posibilidad de incorporar las herramientas de la Web 2.0 y las redes sociales desde una nueva perspectiva formativa, haciendo que la persona adquiera nuevas formas de desenvolverse en el contexto formativo virtual”.

\section{Método}

\section{Propósito}

La investigación tiene como propósito principal establecer el porcentaje de docentes con competencias en tecnologías de información y comunicación y cuántos de éstos la aplican en el aula. Para lograr este objetivo se realizaron diversas actividades investigativas en campo que 
permitieron establecer, a partir del análisis estadístico de la información recopilada por los instrumentos aplicados, el comportamiento de las variables que caracterizan la investigación y así dar cumplimiento al objetivo propuesto.

\section{Muestra}

La muestra de esta investigación estuvo compuesta por 607 personas que hacen parte de las instituciones educativas públicas del municipio de San Juan Girón en Colombia. La totalidad de la muestra está conformada por rectores, coordinadores, profesores y estudiantes. Se aplicó un muestreo probabilístico aleatorio simple entre toda la población objeto de estudio, siendo la muestra productora de información 316 personas correspondientes a 10 rectores, 27 coordinadores, 71 profesores y 208 estudiantes.

\section{Instrumentos}

De acuerdo a las características de la investigación, se aplicaron dos instrumentos de recolección de datos (encuesta y entrevista) para obtener la información de tipo cuantitativa y cualitativa. La encuesta aplicada es anónima y está estructurada en tres partes (la primera de ellas se indaga sobre aspectos relacionados con el perfil personal y professional, en la segunda parte se indaga sobre aspectos que guardan relación con la institución educativa a la cual pertenece, en el tercer aspecto se indaga aspectos relacionados con el uso de las TIC en el aula de clase). La encuesta tiene un total de 43 items cerrados de tipo Likert de 4 alternativas (1. Nunca; 2. Algunas Veces; 3. Casi Siempre; 4. Siempre). Se aplicó el coeficiente de correlación de Pearson para determiner el valor de relación lineal de variables, presentando como resultado valores superiores a 0.86 lo cual indica que existe un grado asociación alto entre las variables de contraste. Los instrumentos de recolección de información fueron validados por juicios de expertos de paises como Chile, España y Colombia, quienes contrastaron el contenido de los instrumentos (gramática, sintaxis, propósito, metodología) con los objetivos de la investigación. Una vez validados los instrumentos, se presenta la versión definitiva que es aplicada en medio físico a la población objeto de estudio. Para la parte cuantitativa se aplicó una entrevista a la población objeto de estudio, donde participaron 12 personas, identificadas como: 3 rectores, 3 coordinadores, 3 profesores y 3 estudiantes. El muestreo aplicado ha sido de tipo opinático o intencional, ya que las personas seleccionadas para la entrevista han sido elegidas con base en la experiencia, conocimiento y punto de vista sobre el tema objeto de interés en la investigación. Las entrevistas se llevan a cabo a través de encuentros personales en las diferentes instituciones educativas. Se realiza un 
análisis de contenido posterior, estableciéndose metacategorías y categorías de definen relaciones para dichas interpretaciones.

\section{Diseño y Procedimiento}

El estudio surge como resultado del análisis de las conclusiones poco favorables obtenidas a partir de la aplicación de lineamientos, que en materia de uso y propiación de las tecnologías de información y comunicación en las instituciones educativas, sugirió el Ministerio de Educación Nacional en Colombia. La investigación involucra dos grandes áreas que guardan relación con la formación docente en tecnologías de información y comunicación que presentan los profesores del municipio de San Juan Girón en Colombia y la aplicación de las mismas al interior de las instituciones educativas. La primera de ellas relacionada con las competencias y conocimientos instrumentales y pedagógicos que tiene la población objeto de estudio sobre las tecnologías de información y comunicación; y la segunda con el grado de aplicación de la mismas al interior de las instituciones educativas. El diseño metodológico es descriptivo de tipo cuantitativo, para lo cual se diseñaron, elaboraron, validaron y aplicaron instrumentos cuantitativos (encuesta) y cualitativos (entrevista) a la población objeto de estudio. Se realizaron análisis estadísticos bidimensionales (tablas de frecuencias, histogramas y gráficos), correlaciones y estudios inferenciales con pruebas no paramétricas y estadísticos de contraste (Chi-Cuadrado de Pearson) con nivel de confianza del 95\%. Para el análisis estadístico de los datos se utilizó la herramienta SPSS (Statistical Package for the Social Sciences). 


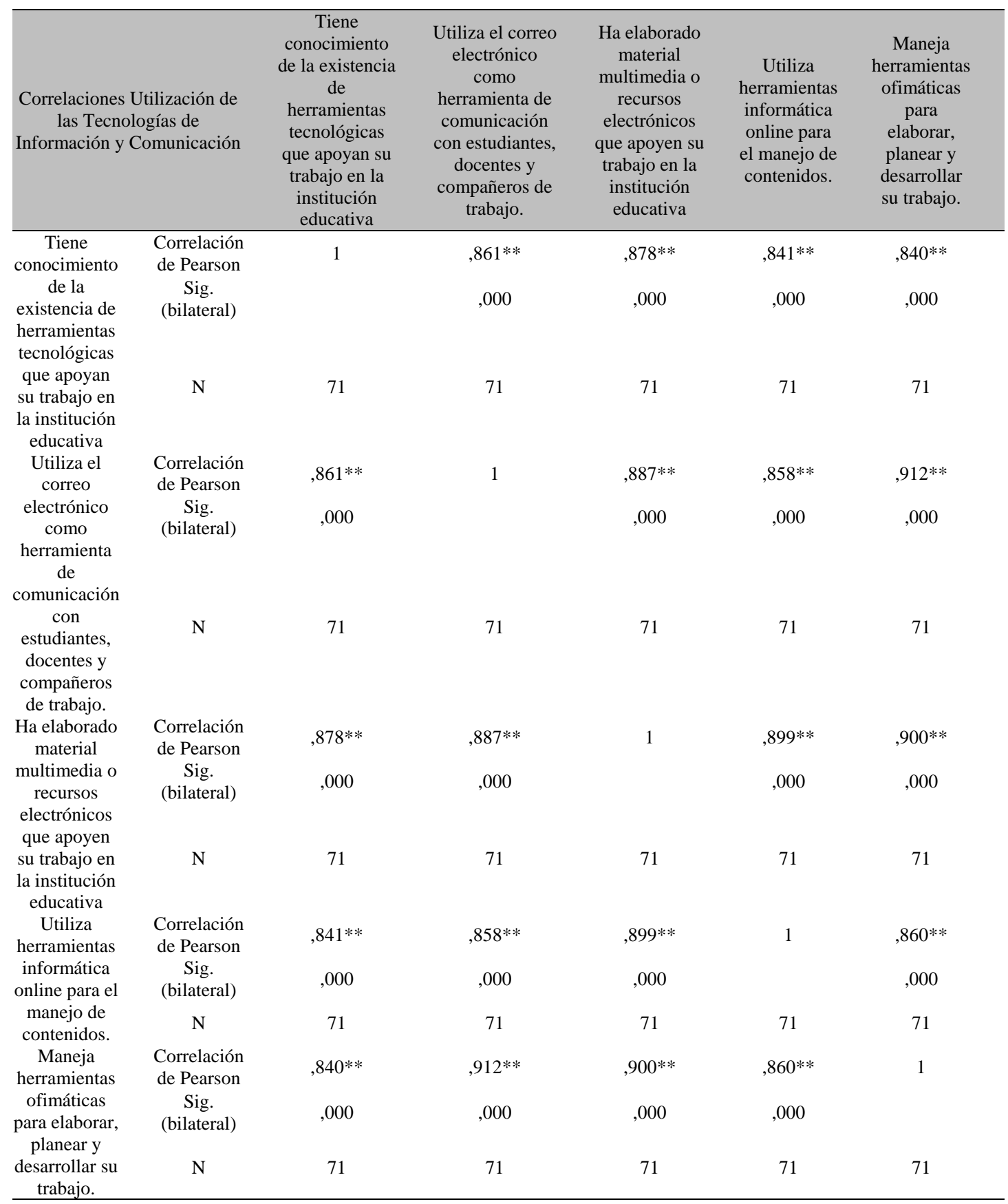

**. La correlación es significativa al nivel 0,01 (bilateral).

Tabla 1. Correlaciones para Utilización de las tecnologías de información y comunicación aplicado a profesores. 


\section{Resultados}

A continuación se describen algunos de los resultados más importantes que guardan relación con el propósito de la investigación:

En general, los resultados muestran que la población estudiada manifiesta en mayor porcentaje (superior al 37.5\%) casi siempre tener conocimiento sobre herramientas tecnológicas que apoyan la labor educativa. Se observa también que son los coordinadores quienes tienen (37\%) mayor conocimiento sobre la existencia de estas herramientas.

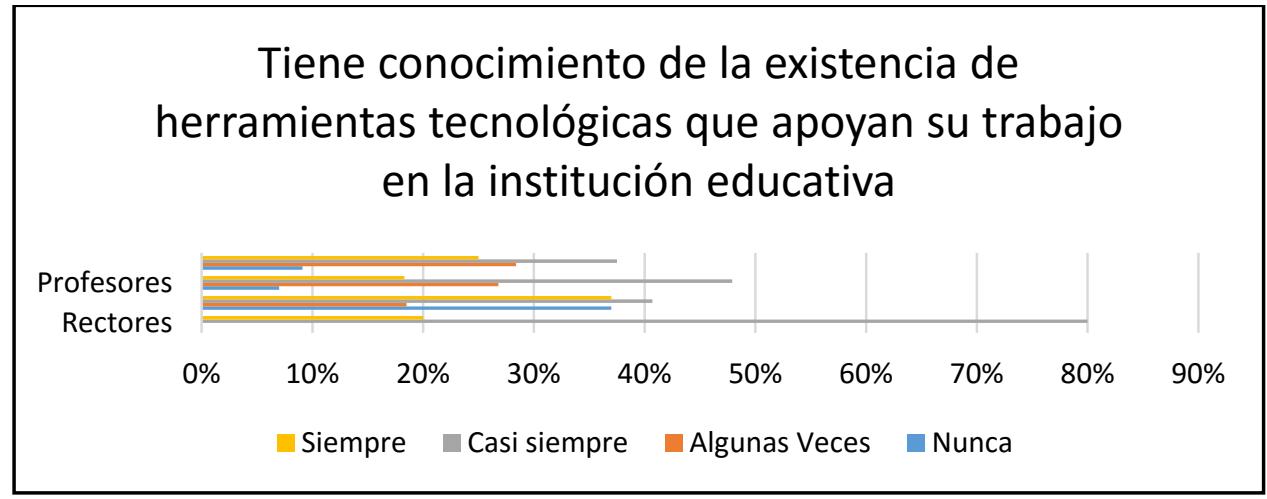

Gráfico 1. Tiene conocimiento de la existencia de herramientas tecnológicas que apoyan su trabajo en la institución educativa

El gráfico 2 muestra que son los estudiantes (19.7\%) quienes siempre incorporan las TIC en sus prácticas. Se observa también que son los profesores (12.7\%) quienes menos las incorporan en sus prácticas educativas.

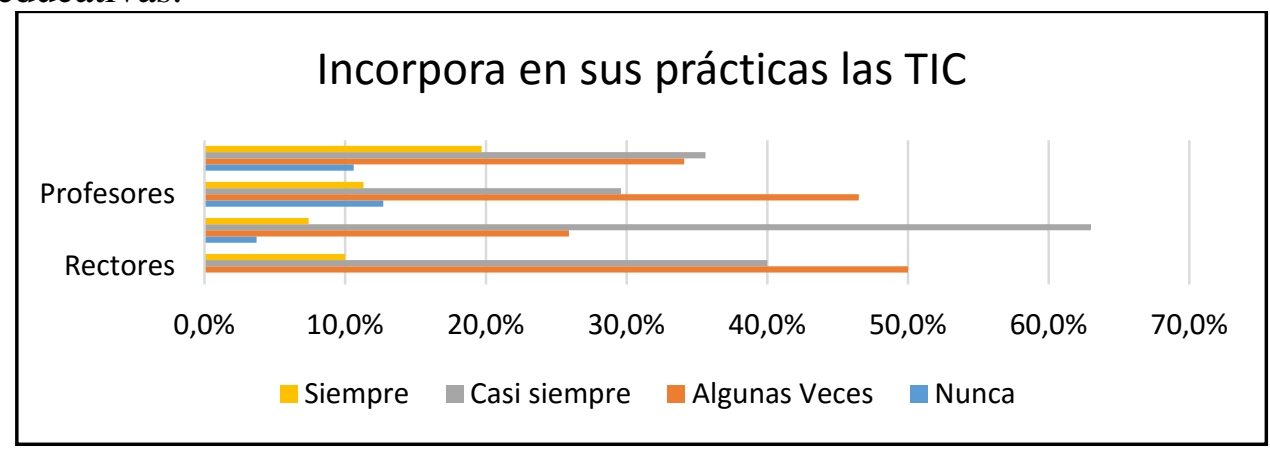

Gráfico 2. Incorpora en sus prácticas las TIC

El gráfico 3 muestra que son los profesores (23.9\%) quienes nunca utilizan el correo electrónico como herramienta de comunicación. Los coordinadores $(55.6 \%)$ manifiestan que casi siempre lo utilizan, mientras que los estudiantes (32.2\%) lo utilizan siempre. 


\section{Utiliza el correo electrónico como herramienta de comunicación con estudiantes, docentes y compañeros de trabajo.}

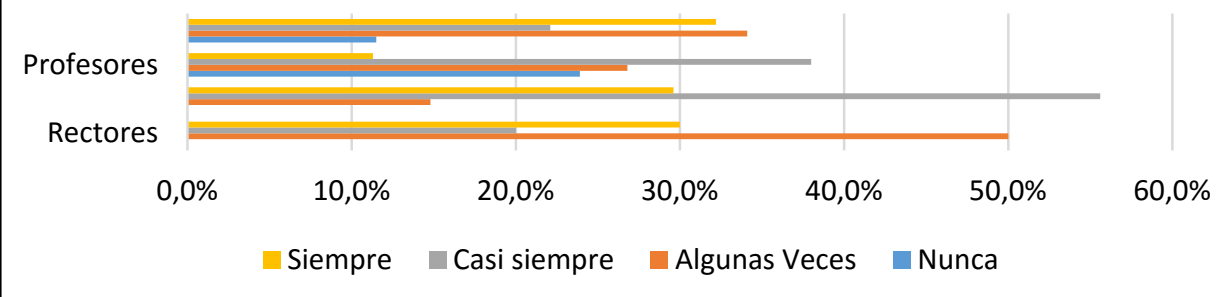

Gráfico 3. Utiliza el correo electrónico como herramienta de comunicación.

El 70\% de los rectores manifiestan que algunas veces han realizado trabajo colaborativo online. Los coordinadores (40.7\%) y los profesores (47.9\%) son quienes nunca han realizado este tipo de actividades. Los estudiantes (36.5\%) nunca han realizado este tipo de actividad. Se observa que solo el $10 \%$ de los rectores realiza trabajo colaborativo.

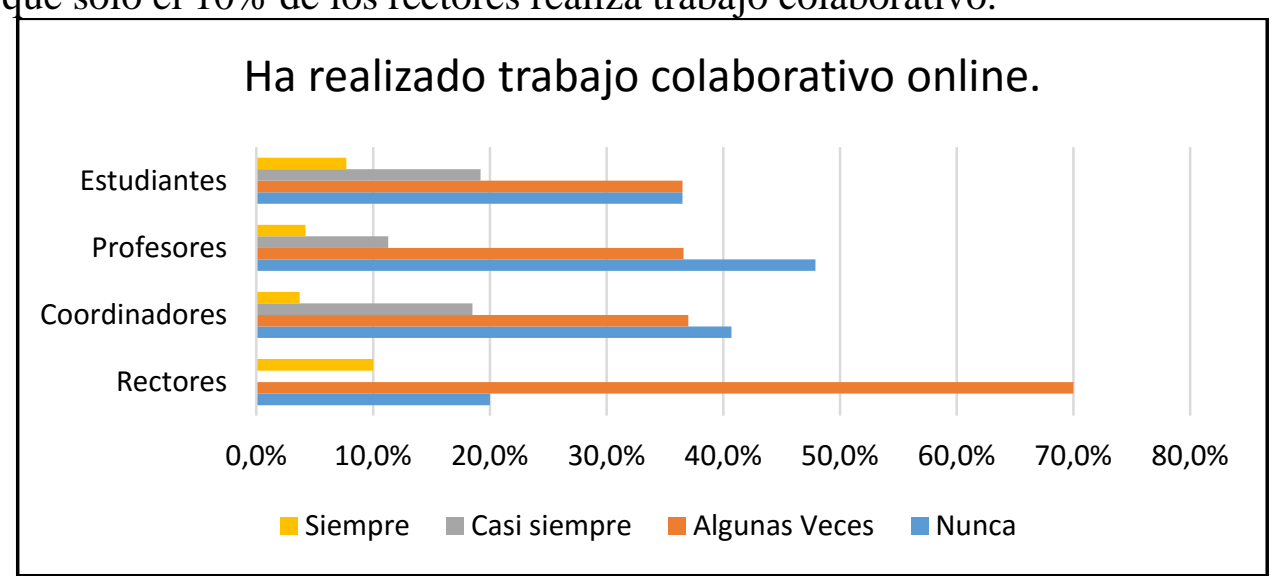

Gráfico 4. Ha realizado trabajo colaborativo online.

Se observa que los rectores (80\%) nunca han elaborado material multimedia o recursos digitales. Los estudiantes (45.2\%) y profesores (42.3\%) manifiestan que algunas veces han elaborado este tipo de recursos de apoyo. Se observa también que la población estudiada no elabora este tipo de recursos. 


\section{Ha elaborado material multimedia o recursos electrónicos que apoyen su aprendizaje en la institución educativa.}

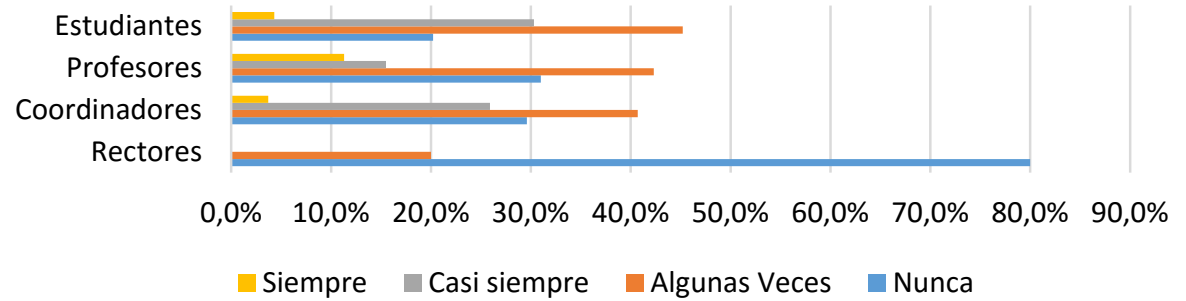

Gráfico 5. Ha elaborado material multimedia o recursos electrónicos.

El 70\% de los rectores afirma que utiliza herramientas informáticas online algunas veces. Los profesores (42.3\%) afirman que nunca utilizan este tipo de herramientas. Se observa que la población estudiada no utiliza herramientas informáticas online para el manejo de contenidos.

\section{Utiliza herramientas informática online para el manejo de contenidos.}

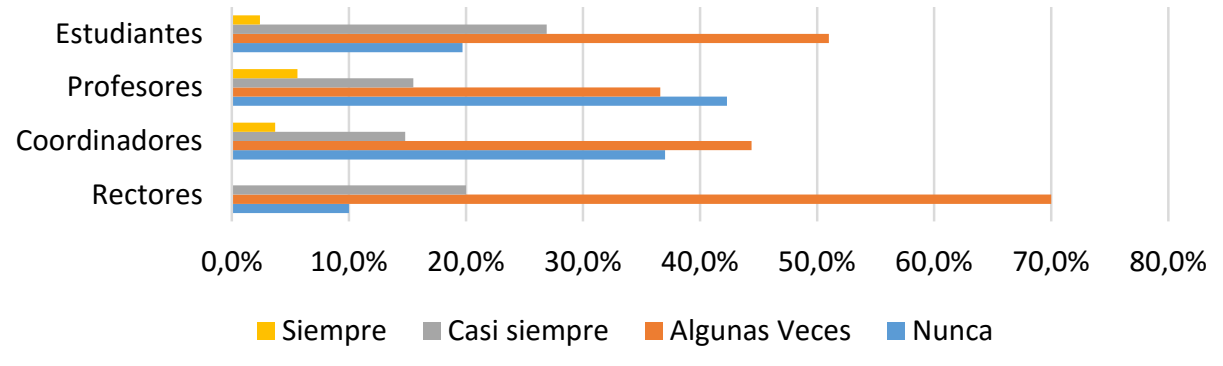

Gráfico 6. Utiliza herramientas informática online para el manejo de contenidos.

\section{Maneja herramientas ofimáticas para elaborar, planear y desarrollar su trabajo.}

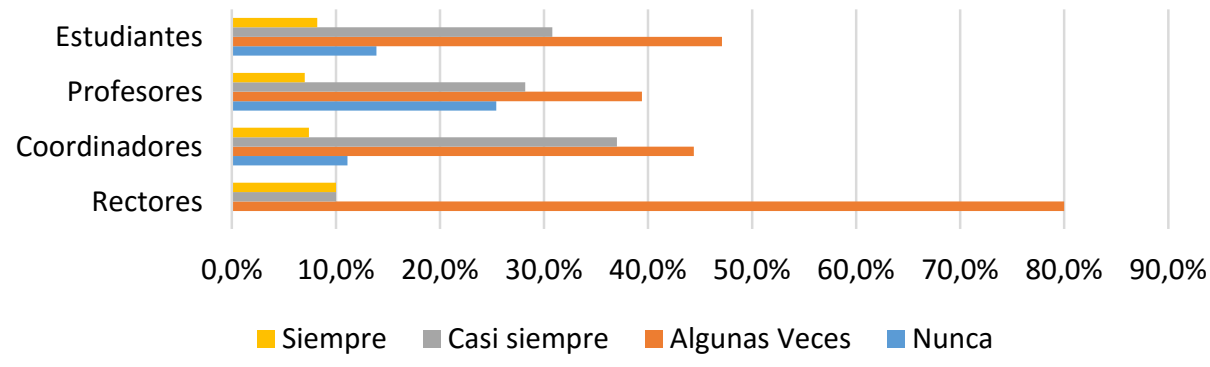

Gráfico 7. Maneja herramientas ofimáticas para elaborar, planear y desarrollar su trabajo. 
Los profesores (25.4\%) manifestaron que nunca utilizan herramientas ofimáticas como apoyo a su trabajo. Se observa que los rectores (80\%) algunas veces las utilizan. Solo el $8.2 \%$ de los estudiantes afirman utilizar siempre este tipo de herramientas como apoyo a su proceso educativo.

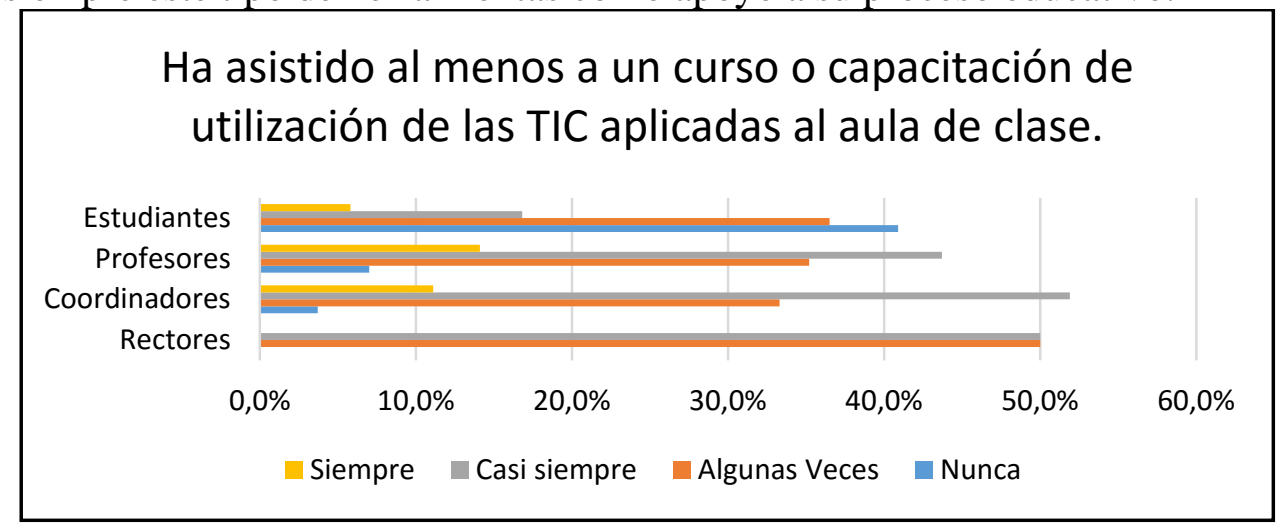

Gráfico 8. Ha asistido a capacitación de utilización de las TIC aplicadas al aula de clase.

Se observa que es muy bajo el porcentaje de la población (14.1\%) que manifiesta haber asistido siempre a procesos de formación en TIC aplicadas al aula de clase. Los estudiantes (40.9\%) manifiestan que nunca han asistido a este tipo de formación. El porcentaje mas alto corresponde a los coordinadores (51.9\%) quienes indican que casi siempre asisten a este tipo de formación.

A continuación se muestran algunos comentarios realizados por la población objeto de estudio, derivados de la aplicación del instrumento de corte cualitativo (entrevista) que permiten complementar las conclusiones.

¿Considera que las tecnologías de información y comunicación permiten
el mejoramiento de la calidad educativa en el municipio de San Juan Girón?

Los rectores entrevistados reconocen que las tecnologías de información y las comunicaciones son de gran importancia para el mejoramiento de la calidad del sector educativo porque permiten generar nuevos aprendizajes a través de la creación de nuevos ambientes académicos involucrando a la comunidad académica. Los coordinadores manifiestan que las TIC fomentan el mejoramiento de la calidad educativa en el municipio, debido a que son una herramienta moderna y actualizada. Las TIC generan alegría y motivan a los estudiantes a generar nuevos aprendizajes. Los profesores manifestaron que las TIC sí permiten el mejoramiento de la calidad educativa porque son una herramienta para mejorar la educación debido a que generan ambientes escolares propicios para la generación y construcción de conocimiento. Los estudiantes manifiestan que las TIC son muy importantes para la educación debido a que actualmente todo está 
relacionado con tecnología. Además manifiestan que la información fluye de mejor manera, permitiendo que la educación sea más dinámica facilitando así el aprendizaje.

¿Qué elementos o variables considera importantes para integrar las tecnologías de información y comunicación en el aula, en el municipio de San Juan Girón?

Los rectores entrevistados consideran que los equipos de cómputo actualizados, la habilidad docente, los ambientes de trabajo adecuados y la formación docente son de gran importancia para integrar las tecnologías de información y comunicación en el aula, en el municipio de San Juan Girón. Los coordinadores dicen que es importante la disposición docente, así como la preparación en temas de TIC y la adquisición de equipos de cómputo, tableros digitales, videobeam y material multimedia adecuado. Se requieren planes de formación y actualización enfocados a docentes, padres, madres de familia y estudiantes. Los profesores dicen que se requieren equipos de cómputo adecuados, además se requiere formación docente en TIC e instalaciones adecuadas, formación permanente de profesores para convertir las TIC en una herramienta de trabajo. Los estudiantes manifiestan que se necesita capacitación a los docentes, equipos tecnológicos, espacios acordes, ambientes adecuados, motivación para aprender y acceso a redes de datos para estar conectados con la tecnología.

¿Por qué razones considera Usted que no se ha integrado en los procesos de enseñanza aprendizaje las tecnologías de información y comunicación en las instituciones educativas en el municipio de San Juan Girón?

Los rectores manifiestan que no se han integrado las TIC en el municipio debido a la falta de liderazgo, a la falta de capacitación y formación docente en TIC, a la poca disponibilidad de espacios y aulas especializadas. También comentan que hacen falta equipos de cómputo adecuados. Los coordinadores dicen que falta una metodología que permita la implementación de las TIC que fomente la transversalización. La falta de espacios, de equipos tecnológicos, material didáctico y la formación docente son otros motivos por los cuales no se están integrando las TIC. Los profesores dicen que no se han integrado las TIC debido a que la capacidad económica de los municipios no lo permite, no existe voluntad política, no hay formación docente en TIC, no se cuenta con espacios suficientes, no se propicia el uso de las TIC. Además señalan que existe un poco de temor a lo nuevo. Los estudiantes responden que no se han integrado las TIC por falta de capacitación a docentes, por falta de espacios adecuados, falta de equipos y de conectividad. 


\section{Conclusion}

Las conclusiones obtenidas a partir del desarrollo de esta investigación deben considerarse de forma prudente pues sus resultados y aportes corresponden estrictamente al contexto particular donde se realizó el estudio (instituciones educativas públicas del municipio de San Juan Girón, Santander Colombia), caracterizandose por limitaciones propias de estudios descriptivos donde intervienen variables particulares y culturales de la población objeto de estudio, lo cual dificulta e imposibilida la replica o generalización de estas contribuciones en otros contextos y realidades educativas diferentes. Sin embargo, sí permiten establecer un marco de referencia base para futuros estudios e investigaciones relacionadas con el tema de la formación docente en tecnologías de información y comunicación en instituciones de educación públicas y la aplicación de las mismas en el aula.

La población estudiada reconoce la importancia de las tecnologías de información y las comunicaciones como herramientas de apoyo a los procesos de enseñanza y aprendizaje, pues permite el mejoramiento de la calidad educativa. Los rectores manifiestan la importancia de tener equipos de computo actualizados, acceso a redes de conocimiento, ambientes adecuados y acordes para el trabajo con tecnología y sobre todo iniciativas de formación y actualización docente en tecnologías de información y comunicación. Los rectores, coordinadores, profesores y estudiantes coinciden en afirmar que no se ha integrado las tecnologías de información y comunicación a los procesos educativos debido a la falta de los elementos mencionados anteriormente.

Se concluye que la población objeto de estudio tiene conocimiento sobre la existencia de herramientas tecnológicas que apoyan la labor educativa, sin embargo son los profesores quienes menos las incorporan a su quehacer pedagógico, lo cual constrasta con los estudiantes quienes las utilizan en mayor porcentaje. Se observa también que la población objeto de estudio carece de planes efectivos de formación pedagógica en tecnologías de información y comunicación, lo cual se evidencia en el bajo porcentaje de uso herramientas informáticas como apoyo a las labores académicas en el aula.

Se observa que la población estudiada no está capacitada en la elaboración de material multimedia y recursos educativos que apoyen el desarrollo de sus actividades. De igual manera tampoco están capacitados para utilizar herramientas informáticas online para el manejo de contenidos. El uso de herramientas ofimáticas para elaborar, planear y desarrollar el trabajo docente se realiza de forma moderada. Se observa también que las actividades de formación tendientes a desarrollar competencias tecnológicas en la población académica no obedecen a un plan de formación lo cual 
imposibilita la aplicación adecuada de las tecnologías de información y comunicación en las instituciones educativas.

Se sugiere el desarrollo y aplicación de un modelo de formación docente en tecnologías de información y comunicación que permita el desarrollo de competencias tecnológicas y su adecuado uso en las aulas al interior de las instituciones educativas, que responda a la realidad educativa de las instituciones del municipio de San Juan Girón, integrando así a toda la comunidad académica.

Finalmente se concluye que en un alto porcentaje los docentes no tienen las competencias necesarias para el uso pedagógico de las TIC como herramienta de apoyo a los procesos educativos lo cual se ve reflejado en la baja aplicación de las mismas en el aula.

\section{References:}

Adell, J. (2010). Escuela 2.0. En C. Barba y S. Capella (coords.). Ordenadores en las aulas: la clave está en la metodología (pp. 19-33). Barcelona: Grao.

Aznar, I. ,Cáceres, M. P., \& Hinojo, F. J. (2005). El impacto de las TICS en la sociedad del milenio: nuevas exigencias de los sistemas educativos ante la “alfabetización tecnológica”. Publicación en línea, 2(4), 177-190.

Barroso, J., Cabero, J., \& Martínez, A. I. V. (2012). La formación desde la perspectiva de los entornos personales de aprendizaje (PLE). Revista Apertura, 4(1).

Barroso, J. (2003). Las nuevas tecnologías de la información y la comunicación y la formación del profesorado universitario. In CIVE 2003 III Congreso Internacional Virtual de Educación.

Cabero, A. J., \& Batanero, J. M. (2014). Una mirada sobre las TIC y la Educación Inclusiva. Comunicación y pedagogía: Nuevas tecnologías y recursos didácticos, (279), 38-42.

Cabero Almenara, J. (2015). Reflexiones educativas sobre las tecnologías de la información y la comunicación (TIC). Revista Tecnología, Ciencia y Educación, volumen (1), 19-27.

Cohen, L., Manion, L., \& Rodríguez, M. A. C. (1990). Métodos de investigación educativa. España. La Muralla.

Del Moral, M. E., y Villalustre, L. (2010). Formación del profesor 2.0. Desarrollo de competencias tecnológicas para la escuela 2.0. Magister, 23, 59-69

Fernandez, F.D, Hinojo, F.J, Aznar, I. (2002). Las actitudes de los docentes hacia la formación en tecnologías de la información y comunicación (TIC) aplicadas a la educación. Contextos educativos: Revista de educación, (5), 253-270. 
Lerma, H. (2009). Metodología de la investigación. Propuesta, anteproyecto, proyecto. Bogotá: Ecoe Ediciones.

Quiroz, J. S. (2012). Estándares TIC para la Formación inicial Docente: Una política pública en el contexto chileno. Education Policy Analysis Archives/Archivos Analíticos de Políticas Educativas, 20, 1-36.

Tamayo y Tamayo, M. (1999). Serie aprender a investigar módulo 5, el proyecto de investigación. Bogotá: Editorial ICFES.

Trujillo, J.M., \& Raso, F. (2010). Formación inicial docente y competencia digital en la convergencia europea (EEES). Enseñanza \& Teaching: Revista Interuniversitaria de Didáctica, 28(1), 49-77.

Trujillo Torres, J. M. (2006). Un nuevo currículum: tecnologías de la información en el aula. Educación y Educadores, 9(1), 161-174.

Trujillo, J.M., Morales, M., Ortiz, A., \& Raso, F. (2015). Percepción del alumnado universitario acerca del uso e integración de las TIC en el proceso educativo de la Facultad de Educación de Granada. Innoeduca: international journal of technology and educational innovation, 1(2), 57-68. 